\title{
The CIDA Schmidt Objective-prism Survey: Fields in Taurus-Auriga and Ophiucus
}

\author{
Cesar Briceño \& Nuria Calvet \\ Centro de Investigaciones de Astronomía, Ap. P. 264, Mérida 5101-A, \\ Venezuela.
}

\begin{abstract}
We have begun a deep objective prism $\mathrm{H} \alpha$ survey of starforming regions using the CIDA $1 \mathrm{~m}$ Schmidt Camera. We report here on the performance of the telescope for this kind of work, based on the study of selected areas in the Taurus-Auriga molecular clouds and the Ophiucus molecular clouds. Up to now seven plates taken in Taurus-Auriga have been visually scanned in a search for pre-main sequence candidates, with a coverage of about 120 square degrees over the whole complex, plus one plate taken in Ophiucus covering the entire cloud $(\approx 18$ square degrees).
\end{abstract}

\section{Searches for Pre-Main Sequence Objects}

The former traditional means of searching for young stars, namely objective prism surveys of $\mathrm{H} \alpha$ emission objects in dark clouds, have been largely abandoned in recent years. New searches for objects with weak emission lines, which would have been missed in $\mathrm{H} \alpha$ objective prism surveys, were undertaken in several star forming regions. Alternative techniques, not biased by spectroscopic measurments, were attempted: proper motions (Hartmann et al. 1991; Gómez et al. 1992), X-ray surveys (Walter \& Kuhi 1981; Feigelson \& Kriss 1981; Feigelson et al. 1987; Walter et al. 1988; Bouvier \& Appenzeller 1992; Strom \& Strom 1993), infrared surveys based on the IRAS Point Source Catalog (Kenyon et al. 1990, Ichikawa \& Nishida 1989) and radio surveys (Leous \& Feigelson 1991).

In spite of the great observational effort invested up to date, surveys of star forming regions so far have yielded relatively few pre-main sequence stars with masses $\mathrm{M} \lesssim 0.4 \mathrm{M}_{\odot}$. However, the initial mass function estimated from field stars suggests that many such low-mass stars should be present (e.g. Kroupa, Tout, \& Gilmore 1991). These low-mass objects are likely to be fainter than could be detected in previous surveys. Moreover, very low-mass stars display prominent $\mathrm{H} \alpha$ emission from their birth all the way to the main sequence, so that observational biases against finding weak-emission stars are relatively unimportant.

\section{Observations and Results}

The $\mathrm{H} \alpha$ emission survey has been carried out with the $\mathrm{f} / 3,100 / 150 \mathrm{~cm}$ Schmidt Camera at Observatorio de Llano del Hato, Mérida, Venezuela, using equipment described in Briceño et al. (1993). The spectra were widened by about $0.1 \mathrm{~mm}$, 
and the plates have been scanned visually. The characteristics of the observational material used in this work are described in Table I. Prism or direct plates are distinguished by a "P" or a " $D$ " next to the plate number.

TABLE I Observational Material

\begin{tabular}{|c|c|c|c|c|c|c|c|}
\hline Field & Plate No. & $\alpha_{0}(1950)$ & $\delta_{o}(1950)$ & $\begin{array}{l}\text { Exp. } \\
(\min )\end{array}$ & Filter & Date & Emulsion \\
\hline $\begin{array}{l}\text { TAU1 } \\
\text { TAU1 } \\
\text { TAU1 } \\
\text { TAU4 } \\
\text { TAU5 } \\
\text { TAU2 } \\
\text { TAU2 } \\
\text { TAU6 } \\
\text { TAU7 } \\
\text { TAU3 } \\
\text { TAU3 } \\
\text { OPH1 }\end{array}$ & $\begin{array}{l}1197 \mathrm{P} \\
1231 \mathrm{P} \\
1314 \mathrm{P} \\
1096 \mathrm{P} \\
1198 \mathrm{D} \\
1228 \mathrm{P} \\
1215 \mathrm{P} \\
1216 \mathrm{P} \\
1309 \mathrm{P} \\
1268 \mathrm{P}\end{array}$ & $\begin{array}{c}4^{h} 19^{m} 16^{\prime} \\
4^{h} 28^{m} 00^{A} \\
4^{h} 4^{m} 00^{\circ} \\
4^{h} 35^{m} 00^{\circ} \\
4^{h} 35^{m} 00^{\circ} \\
4^{h} 44^{m} 00^{\circ} \\
4^{h} 45^{m} 00^{\circ} \\
5^{h} 04^{m} 00^{\prime} \\
5^{h} 04^{m} 00^{\circ} \\
16^{h} 28^{m} 00^{\circ}\end{array}$ & $\begin{array}{l}+27^{\circ} 47^{\prime} 57^{\prime \prime} \\
+28^{\circ} 01^{\prime} 17^{\prime \prime} \\
+28^{\circ} 01^{\prime} 17^{\prime \prime} \\
+30^{\circ} 28^{\prime} 00^{\prime \prime} \\
+28^{\circ} 50^{\prime} 00^{\prime \prime} \\
+24^{\circ} 38^{\prime} 01^{\prime \prime} \\
+24^{\circ} 38^{\prime} 01^{\prime \prime} \\
+34^{\circ} 03^{\prime} 00^{\prime \prime} \\
+25^{\circ} 05^{\prime} 00^{\prime \prime} \\
+25^{\circ} 40^{\prime} 00^{\prime \prime} \\
+25^{\circ} 40^{\prime} 00^{\prime \prime} \\
-23^{\circ} 47^{\prime} 00^{\prime \prime}\end{array}$ & $\begin{array}{l}20 \\
60 \\
30 \\
90 \\
120 \\
90 \\
30 \\
90 \\
90 \\
90 \\
15 \\
120\end{array}$ & $\begin{array}{l}- \\
- \\
\text { OG590 } \\
\text { OG590 } \\
\text { OG590 } \\
\text { OG590 } \\
\text { OG590 } \\
\text { OG590 } \\
\text { OG590 } \\
\text { OG590 } \\
\text { OG590 } \\
\text { OG590 }\end{array}$ & $\begin{array}{l}01 / 26 / 87 \\
12 / 19 / 90 \\
10 / 06 / 91 \\
09 / 01 / 92 \\
30 / 10 / 92 \\
12 / 20 / 90 \\
10 / 06 / 91 \\
08 / 01 / 92 \\
09 / 01 / 92 \\
12 / 09 / 91 \\
09 / 30 / 92 \\
09 / 03 / 92\end{array}$ & $\begin{array}{l}\text { III-aO } \\
\text { III-aF } \\
\text { III-aF } \\
\text { III-aF } \\
\text { III-aF } \\
\text { III-aF } \\
\text { III-aF } \\
\text { III-aF } \\
\text { III-aF } \\
\text { III-aF } \\
\text { III-aF } \\
\text { III-aF }\end{array}$ \\
\hline
\end{tabular}

We tested the performance of the telescope with selected fields in Taurus (Briceño et al. 1993). Of the 68 known stars in the calibration area, $60 \%$ of the objects with $\mathrm{W}(\mathrm{H} \alpha)>10 \AA$ were detected, while $80 \%$ of the stars with $\mathrm{W}(\mathrm{H} \alpha)>50 \AA$ were detected. Stars with $\mathrm{W}(\mathrm{H} \alpha)<10 \AA$ were detected only when the line-to-continuum contrast was favorable (such as J 4423 and JH 223, with $\left.\mathrm{W}_{\lambda}(\mathrm{H} \alpha) \sim 5 \AA\right)$. Stars brighter than $\mathrm{V} \approx 13.5$ were not detected. Fig.1 summarizes these results and gives a crude estimate of the survey limits.

A spectral classification was developed in the red part of the spectrum for the resolution of the instrument. Comparison of the red spectral types with those published generally give agreement within two subclasses.

In fields TAU1 and TAU2, and in a third field that included L1544 (TAU3 in Table I), $37 \mathrm{H} \alpha$ emission stars that did not appear in the lists mentioned were found. The pre-main sequence nature of 12 objects was confirmed from MMT spectra (Briceño et al. 1993). These stars together with the new candidates from fields TAU4, TAU5, and TAU6 are shown in Fig. 3, projected against the contours of ${ }^{12} \mathrm{CO}$ emission from Ungerechts and Thaddeus (1987). Candidates in field TAU7 were checked for $\mathrm{Li} 6707 \AA$ in spectra taken at the MMT, none of them showing any absorption at this wavelength that would indicate their young nature.

The plate taken in Ophiucus (Field OPH1) turned up many emission objects. We detected about $90 \%$ of the Wilking et al. (1987) survey objects, plus all Herbig \& Bell (1988) catalog stars in the area covered by our plate and within the survey limits (Fig. 1). The remaining objects are shown as new $\mathrm{H} \alpha$ emission objects in Fig. 2, projected against the ${ }^{13} \mathrm{CO}$ contours from Loren (1989) and infrared sources from Ichikawa \& Nishida (1989).

\section{Discussion}

The results obtained so far indicate that we are detecting objects with moderate or strong $\mathrm{H} \alpha$ emission $\left(\mathrm{W}_{\lambda}(\mathrm{H} \alpha) \gtrless 10\right)$ down to about $\mathrm{V} \approx 18$, with a completeness factor of over $60 \%$ (Fig. 1). Stars detected at this level cannot be given a spectral type but can be identified as emission objects if $\mathrm{H} \alpha$ is present. Taking 


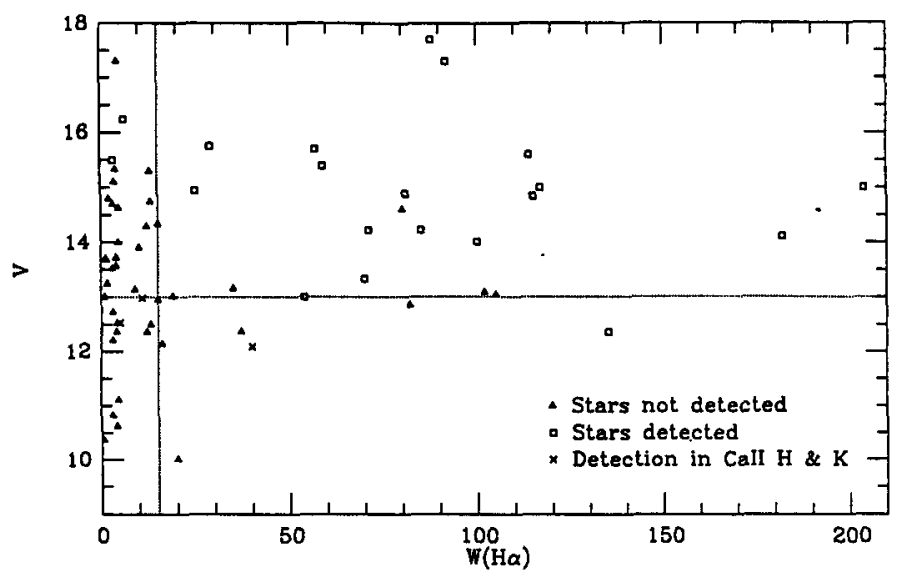

Figure 1. Data from the three calibration fields in Taurus indicating the survey detection limits in both visual magnitude and $\mathrm{H} \alpha$ equivalent width.

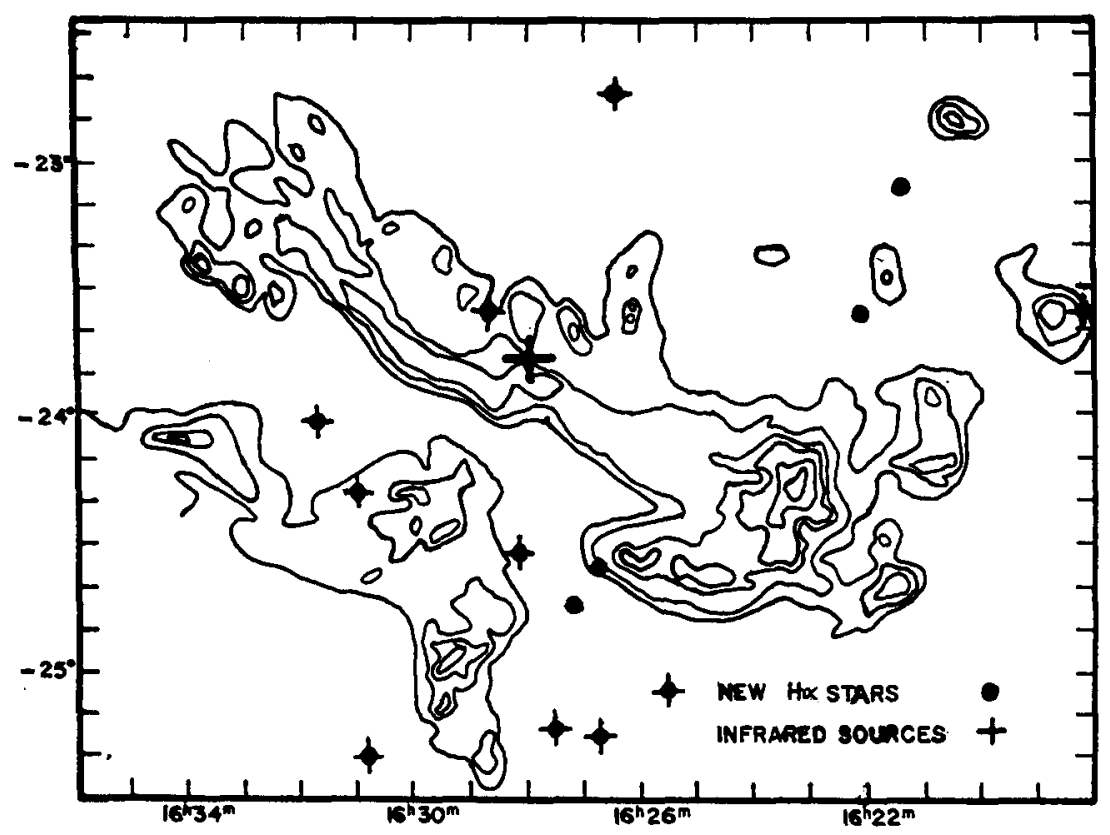

Figure 2. New $\mathrm{H} \alpha$ emission stars detected in Ophiucus, projected against the ${ }^{13} \mathrm{CO}$ contours from Loren (1989) and superposed with IRAS sources from Ichikawa \& Nishida (1989). 


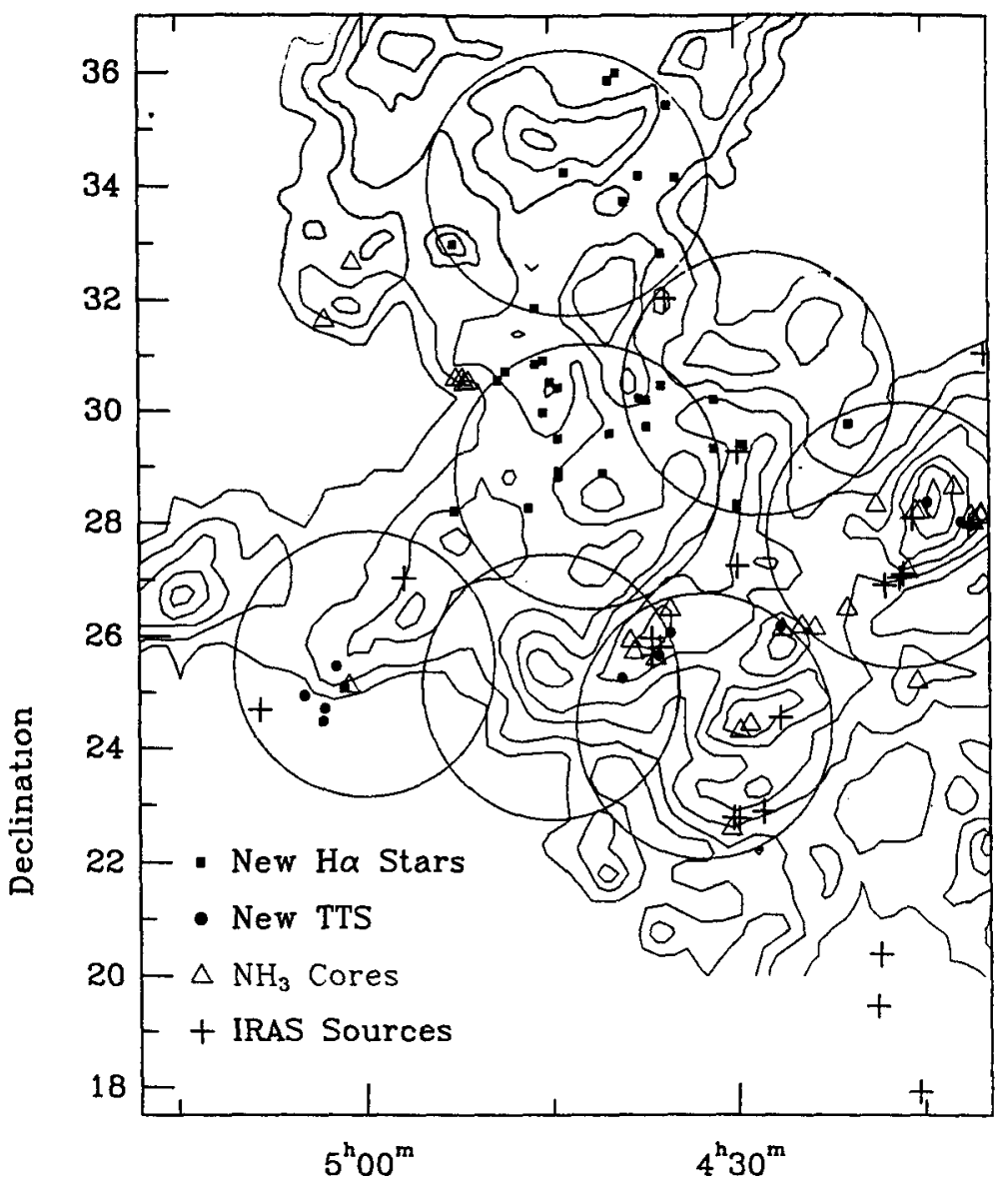

Right Ascension

Figure 3. Fields searched in Taurus-Auriga. The new T Tauri stars reported in Briceño et al. (1993) are indicated, together with new $\mathrm{H} \alpha$ emission candidates. Contours are the ${ }^{12} \mathrm{CO}$ distribution from Ungerechts \& Thaddeus (1987). Also indicated are IRAS Sources from Kenyon et al. (1990) and $\mathrm{NH}_{3}$ cores from Myers \& Benson (1983). 
into account seeing variations typical among different nights at Llano del Hato Observatory, we can estimate an average dispersion of about 1 magnitude in the faint limit of different plates. The spectral typing is thus limited to objects brighter than $\mathrm{V} \approx 17$.

Of the 12 new pre-main sequence stars, 9 are classical T Tauri stars (CTTS), with $\mathrm{W}_{\lambda}(\mathrm{H} \alpha) \gtrsim 10 \AA$. Jones \& Herbig (1979) derived a model for the expected number of emission line stars in the Taurus clouds and compared the numbers with those known at the time. They found good agreement with the observations, assuming that no emission stars fainter than $M_{p g}=+9.5$ existed. The new stars discovered, together with those found in recent surveys, have increased considerably (given the small number statistics of the cloud) the number of faint emission stars, which implies that the cut off in magnitude should be fainter. Of the 14 candidates in Ophiucus, 10 overlap IRAS sources from Ichikawa \& Nishida (1989), which points to the fact that they probably are pre-main sequence objects.

At the CIDA Schmidt telescope, a new dedicated Tektronix $2048 \times 2048$ is scheduled to be commissioned during late 1994-early 1995 . When installed, the full field will be about $3 / 4^{\circ}$ square with a scale of $0.8 \mathrm{arcsec} / \mu \mathrm{m}$. The telescope will neverthless retain its capability to use plates. Future work foresees conducting deeper searches in limited areas and obtaining CCD objective prism spectra for better signal-to-noise data of candidates whose emission nature couldn't be well determined from the plate material. Slit spectra of the candidates will continue to be taken as possible, and follow up photometric observations are also planned.

\section{Acknowledgements}

C. B. and N. C. would like to thank J. Stock for his help in setting the red spectral classification in objective prism spectra and in the general use of the prism. C. Abad kindly introduced C. B. to the use of the astrometric programs developed by the Astrometry Group at CIDA. We also acknowledge the support of the Technical Department of CIDA and of the night operators during the development of this project.

\section{References}

Bouvier J. \& Appenzeller I., 1992, A\&AS, 92, 481

Briceño C., Calvet N., Gómez M., Hartmann L. W., Kenyon S. J., \& Whitney B. A., 1993, PASP, 105, 686

Feigelson E. D. \& Kriss G. A., 1981, ApJ, 248, L35

Feigelson E. D., Jackson J. M., Mathieu R. D., Myers P. C. \& Walter F. M., 1987, AJ, 94, 1251

Gómez M., Jones B. F., Hartmann L. W., Kenyon S. J., Stauffer J. R., Hewett R. \& Reid I. N., 1992, AJ, 104, 762

Hartmann L. W., Jones B. F, Stauffer J. R. \& Kenyon S. J., 1991, AJ, 101, 1050 Herbig G. H. \& Bell K., 1988, Lick Obs. Bull. Ser., No. 1111 
Ichikawa T. \& Nishida M., 1989, AJ, 97, 1074

Jones B. F. \& Herbig G. H., 1979, AJ, 84, 1872

Kenyon S. J., Hartmann L. W., Strom K. M. \& Strom S.E., 1990, AJ, 99, 869

Kroupa P., Tout C. A. \& Gilmore G., 1991, MNRAS, 251, 293

Leous J. A. \& Feigelson E. D., 1991, ApJ, 379, 683

Loren R.B., 1989, ApJ, 338, 902

Myers P. C. \& Benson P.J., 1983, ApJ, 266, 309

Ungerechts H. \& Thaddeus P., 1987, ApJS, 63, 645

Walter F. M. \& Kuhi L.V., 1981, ApJ, 250, 254

Walter F. M., Brown A., Mathieu R. D., Myers P. C. \& Vrba F. V., 1988, AJ, 96, 297

Wilking B. A., Schwartz R. D. \& Blackwell J. H., 1987, AJ, 94, 106

\section{Discussion}

MacConnell: It is essential to follow up the $\mathrm{H} \alpha$ emission objects with slit observations. Some years ago, I worked with George Coyne to 'clean-up' the Vatican catalogue of possible Be stars, many of which, in fact did not have emission, but were $\mathrm{M}$ giants with $\mathrm{TiO}$ band-heads that were mistaken for $\mathrm{H} \alpha$ emission on the low-resolution plates.

Briceño: I agree with you on this issue; this is why we are conscious that the survey with the Schmidt is only the starting point for generating candidate lists that will then be observed for obtaining slit spectra of suitable resolution, not only to confirm the $\mathrm{H} \alpha$ emission nature but to look for the $\mathrm{Li} 6706 \AA$ line which is the signature of the pre-main sequence star. Only then do we consider our work in a given field to be ready for follow-up photometric observations (both optical and infrared) of the newly identified pre-main sequence objects.

Hidayat: Some non- $\mathrm{H} \alpha$ stars in the southern Coalsack region show variable CaII in emission. Would you comment on that?

Briceño: Usually pre-main sequence stars with CaII $\mathrm{H} \& \mathrm{~K}$ emission show little or weak $\mathrm{H} \alpha$ emission, well below the detection limit of low dispersion $\mathrm{H} \alpha$ objective-prism surveys, so they are missed in surveys like the one we are conducting. Nevertheless, most young low-mass stars display prominent $\mathrm{H} \alpha$ emission, almost all the way down to the main sequence. In addition, these stars are very faint, so our technique is particularly well suited to detecting them. Observational biases against finding weak $\mathrm{H} \alpha$ emission stars are relatively unimportant. 